Relations industrielles

Industrial Relations

\title{
Analysing Jobs, by M.B. Youngman, R. Oxtoby, J.D. Monk and J. Heywood, Epping, Essex, Eng., Gower Press, 1978, 157 pp.
}

\section{Gilles Ferland}

Volume 35, numéro 3, 1980

URI : https://id.erudit.org/iderudit/029113ar

DOI : https://doi.org/10.7202/029113ar

Aller au sommaire du numéro

Éditeur(s)

Département des relations industrielles de l'Université Laval

ISSN

0034-379X (imprimé)

1703-8138 (numérique)

Découvrir la revue

Citer ce compte rendu

Ferland, G. (1980). Compte rendu de [Analysing Jobs, by M.B. Youngman, R. Oxtoby, J.D. Monk and J. Heywood, Epping, Essex, Eng., Gower Press, 1978, 157 pp.] Relations industrielles / Industrial Relations, 35(3), 613-613.

https://doi.org/10.7202/029113ar

Tous droits réservés (C) Département des relations industrielles de l'Université Laval, 1980
Ce document est protégé par la loi sur le droit d'auteur. L'utilisation des services d'Érudit (y compris la reproduction) est assujettie à sa politique d'utilisation que vous pouvez consulter en ligne.

https://apropos.erudit.org/fr/usagers/politique-dutilisation/ 
une seconde étape, elle décidait du taux de l'augmentation intermédiaire. Par hypothèse, lesg2 étapes étaient considérées comme statistiqape, elle décidait du taux de l'augmentation intermédiaire. Par hypothèse, les 2 étapes étaient considérées comme statistiquement indépendantes.

Parmi la gamme des variables possibles qui auraient pu influencer les décisions de la Commission (indicateurs, augmentation négociée, secteur d'activité, région, professions...), les résultats indiquent «que les seules variables qui sont constamment des déterminants significatifs de cette décision sont l'indicateur et le taux d'augmentation négocié».

L'étude démontre aussi qu'à mesure que progressait le programme anti-inflationniste les caractéristiques particulières à chaque cas ont joué un rôle de moins en moins important dans la décision de la Commission.

Dans un contexte d'inflation chronique qui accentue le danger de retour à des mécanismes de contrôle des prix et des revenus, des études de ce genre sont appropriées.

\section{Conseil du Trésor}

\section{Jean-Marc ROBICHAUD}

Gouvernement du Québec

Analysing Jobs, by M.B. Youngman, R. Oxtoby, J.D. Monk and J. Heywood, Epping, Essex, Eng., Gower Press, 1978, $157 \mathrm{pp}$.

L'analyse des emplois est une technique qui peut être utile dans plusieurs domaines de la direction du personnel et des relations professionnelles: organisation du travail, recrutement, sélection, placement, formation, promotion, amélioration des conditions de travail, évaluation des emplois, négociations collectives, règlement des griefs, etc. Cette technique connue depuis des décennies a surtout été utilisée jusqu'à maintenant pour l'étude du contenu des emplois de métier, de production et de bureau. Rarement a-t-elle été adaptée à l'analyse des emplois professionnels et de direction sous prétexte que ces emplois ne se prêtaient guère à une telle analyse à cause du caractère complexe des tâches qu'ils comportent. C'est cette lacune qu'ont voulu combler les auteurs dans leur ouvrage Analysing Jobs.

Bien que l'étude ne porte que sur l'analyse des emplois d'ingénieurs et ce, dans le but spécifique de définir des objectifs de formation pour cette catégorie d'employés, le processus d'analyse élaboré par les auteurs peut être appliqué à d'autres catégories d'emplois tout comme les résultats de l'analyse peuvent également être utilisés à des fins autres que celles de la formation du personnel.

La méthode mise au point dans cet ouvrage consiste principalement à choisir dans un premier temps un échantillon représentatif des postes de travail à analyser, à interviewer ensuite à l'aide d'entrevues semi-structurées les titulaires de ces postes dans le but de leur fair` décrire leur travail et enfin à établir une liste exhaustive des tâches accomplies. Une copie de cette liste est ensuite transmise à tous les autres travailleurs visés par l'enquête et chacun doit y pointer les tâches qu'il exécute dans l'exercice de ses fonctions. Une fois les résultats compilés, le regroupement des postes de travail en emplois est fait par la méthode de classification de Ward qui consiste essentiellement à faire une comparaison par paires de tous les postes analysés, à calculer le nombre de tâches différentes pour chaque paire de postes comparés, à reporter graphiquement les résultats et à déterminer les postes qui représentent suffisamment de similitudes pour être regroupés sous un même titre.

Le processus d'analyse des emplois décrit ci-haut est ensuite complété par une étude portant sur les attitudes des ingénieurs à l'égard de leur travail ainsi que sur leur expérience de travail.

Analysing Jobs est un ouvrage qui peut être un outil précieux non seulement pour les spécialistes en analyse des tâches et en évaluation des emplois, mais aussi pour les gestionnaires intéressés par les problèmes d'organisation du travail et de formation du personnel.

Gilles FERLAND

Université Laval 